\title{
A closer look at dynamic speckles and the use of their fine-structure for object measurements
}

\author{
Hanson, Steen Grüner; Iversen, Theis F.Q.; Jakobsen, Michael Linde
}

Published in:

Proceedings of SPIE - The International Society for Optical Engineering

Link to article, DOI:

$10.1117 / 12.870936$

Publication date:

2010

Document Version

Early version, also known as pre-print

Link back to DTU Orbit

Citation (APA):

Hanson, S. G., Iversen, T. F. Q., \& Jakobsen, M. L. (2010). A closer look at dynamic speckles and the use of their fine-structure for object measurements. Proceedings of SPIE - The International Society for Optical Engineering, 7387, 73870W. https://doi.org/10.1117/12.870936

\section{General rights}

Copyright and moral rights for the publications made accessible in the public portal are retained by the authors and/or other copyright owners and it is a condition of accessing publications that users recognise and abide by the legal requirements associated with these rights.

- Users may download and print one copy of any publication from the public portal for the purpose of private study or research.

- You may not further distribute the material or use it for any profit-making activity or commercial gain

- You may freely distribute the URL identifying the publication in the public portal 


\title{
A closer look at dynamic speckles and the use of their fine-structure for object measurements
}

\author{
Steen G. Hanson ${ }^{1}$ Theis F.Q. Iversen ${ }^{2}$ and Michael Linde Jakobsen ${ }^{1}$ \\ 1. DTU Fotonik, Department of Photonics Engineering, Technical University of Denmark, \\ P.O. Box 49, DK-4000, Roskilde, Denmark \\ 2. OPDI Technologies A/S, Denmark,
}

\begin{abstract}
The possibility to "dress up" the speckles and thereby providing them with a fine structure will be discussed. As these speckles arise from scattering off solid targets, the dynamics of the speckles and their inherent fine structure might vary, providing information on different aspects of the surface displacement. This is achieved by illuminating the object with structured light, and observing the speckle field as it passes an optical system. In this way, simultaneous measurement of displacement (e.g. 2-D) and rotation can be performed. The application of this concept with a system based on spatial filtering velocimetry and ordinary speckle correlation will be discussed.
\end{abstract}

Key words: speckle dynamics, velocity measurement, speckled speckles

\section{INTRODUCTION}

When coherent light is scattered off a rough surface, the resulting field in the observation after passage of an optical system has to be found by adding up the field contribution from the entire surface. In general, this will result in a highly intensity modulated pattern, named speckles. This issue has been frequently addressed and several comprehensive treatment have been offered ${ }^{1}$. These evaluations are usually based on illuminating the object with a single Gaussian beam. In the present treatment we will review the speckle properties when the object is illuminated with an array of beams, and specifically when only two beams of varying mutual position and angle of incidence illuminate the object ${ }^{2,3}$. Investigations of this phenomena giving rise to a fine structure in the speckle field, named "compound speckles", have subsequently been addressed. A description of the three dimensional structure of the speckle field by crossing beams have been given ${ }^{4}$.

The dynamics of speckle patterns for probing object dynamics have been analyzed by optically correlating speckle images before and after a lateral translation ${ }^{5,6}$ and for probing rotational displacements ${ }^{7}$. The associated accuracy and uncertainties have been analyzed in case of intensity correlation between subsequent recordings ${ }^{8}$.

The motivation for this investigation is the application for miniaturized optical sensors for displacement measurement, being it measurement of rotation, linear displacement or the associated vibrations. In order to meet the requirements for miniaturized sensors, we employ spatial filtering velocimetry ${ }^{9-11}$. As the systems we investigate address the consumer market, laser eye safety is a second motivation for splitting up of the illuminating beam ${ }^{12}$.

Speckle 2010: Optical Metrology, edited by Armando Albertazzi Goncalves Jr., Guillermo H. Kaufmann, Proc. of SPIE Vol. 7387, 73870W · (c) 2010 SPIE · CCC code: 0277-786X/10/\$18 - doi: 10.1117/12.870936 
The theoretical investigation will follow the path presented in a previous paper ${ }^{13}$. There, the formalism based on complex $A B C D$-matrices ${ }^{14}$ was combined with the statistical properties for speckle statistics ${ }^{15}$. After revisiting the theoretical evaluation, the main results will be presented, with special emphasis on the dynamical behavior (speckle size, velocity and decorrelation) for the three generic system, viz. free space propagation, imaging and Fourier transform. The applications being for miniaturized optical sensors, the implications for establishing systems being sensitive to only displacement or rotation will be stressed. Following this, a conclusion will sum up the findings.

\section{THEORETICAL DESCRIPTION}

We will adhere to the formalism of complex $A B C D$-matrices, here with the restrictions that

- the input- and output plane are placed in a medium with the same refractive indices

- the optical system is rotationally symmetric

- the optical elements consist of free space, mirrors, parabolic reflectors, free space and apertures, which are Gaussian apodized

- the statistics of the scattering surface is isotropic and space independent

- the statistics for the field in the output plane is circularly symmetric Gaussian

The (scalar) electric field, $U(\mathbf{p}, t)$, in the observation is given by

$$
U(\mathbf{p}, t)=\int G(\mathbf{r}, \mathbf{p}) U_{r e f}(\mathbf{r}, t) d \mathbf{r},
$$

where $G(\mathbf{r}, \mathbf{p})$ is the Green's function connecting the observation plane with the time dependent field(s) in the object plane, $U_{r e f}(\mathbf{r}, t)$. The Green's function is in the matrix formalism given by

$$
G(\mathbf{r}, \mathbf{p}) \propto \exp \left[\frac{i k}{2 B}\left(A \mathbf{r}^{2}-2 \mathbf{r} \cdot \mathbf{p}+D \mathbf{p}^{2}\right)\right] .
$$

Here, the effect of the entire train of optical elements making up the optical system connecting the object with the observation plane is embedded in the three complex parameters $A, B$ and $D$. The scattering surface is assumed to give rise to a fully developed speckle field, being it based on transmission or reflection. This means that the phase change between two adjacent positions is uncorrelated, and further that the probability distribution for the phase is uniform from 0 to $2 \pi$. Displacement of the object a distance $\Delta \mathbf{r}$ will make this structure move as a frozen pattern without any spatial decorrelation. This means that we are only considering rigid structures. The scattered field leaving the object can then be written

$$
U_{r e f}(\mathbf{r}, t)=\Psi(\mathbf{r}, t) U_{i n c}(\mathbf{r}),
$$

where the time -independent field(s) incident on the object is given by $U_{i n c}(\mathbf{r})$ and the phase function of the object giving rise to fully developed speckles has the following properties:

$$
\left\langle\Psi\left(\mathbf{r}_{1}, t\right) \Psi *\left(\mathbf{r}_{2}, t+\tau\right)\right\rangle \propto \delta\left(\mathbf{r}_{1}-\mathbf{r}_{2}+\mathbf{v} \tau\right) .
$$

Here angular brackets mean "ensemble average" and $\boldsymbol{v}$ is the transverse velocity of the object. No longitudinal object velocity is assumed present. Neither have we assumed any spatial reflective intensity variations, nor have we included the overall reflective/transmissive factors as we are solely interested in the previously described parameters. We now posses the necessary tools for tracking the field from the object to the observation plane.

Calculation of the space- and time-lagged crosscovariance of the intensity before and after an object displacement will provide us with all the information necessary to describe the speckle behavior in the observation plane. The space- and time-lagged intensity covariance is given by:

$$
\Gamma(\Delta \mathbf{p}, \tau)=\langle I(\mathbf{p}, t) I(\mathbf{p}-\Delta \mathbf{p}, t-\tau)\rangle-\langle I(\mathbf{p}, t)\rangle\langle I(\mathbf{p}-\Delta \mathbf{p}, t-\tau)\rangle,
$$


and on the optical axis in normalized form:

$$
\begin{aligned}
& \Gamma_{N}(\Delta \mathbf{p}, \tau) \equiv \frac{\Gamma(\Delta \mathbf{p}, \tau)}{\Gamma(0,0)}=|\gamma(\Delta \mathbf{p}, \tau)|^{2} \text { where } \\
& \gamma(\Delta \mathbf{p}, \tau)=\frac{\left\langle U(\mathbf{0}, t) U^{*}(\Delta \mathbf{p}, t-\tau)\right\rangle}{\left\langle U(\mathbf{0}, t) U^{*}(\mathbf{0}, t)\right\rangle}
\end{aligned}
$$

Arriving at the last expression in Eq. (6), we have taken advantage of the field obeying Gaussian statistics and being circularly symmetric, i.e. the Siegert relation. The initial task of calculating a fourth-order field correlation function, Eq. (5), has now been reduced to deriving a second order field correlation function in the output plane.

Had the input field $U_{0}(\mathbf{r})$ consisted of a single Gaussian beam, the three desired parameters, namely the average speckle size, the speckle velocity and their decorrelation length have previously been found ${ }^{16}$. Here, our goal is to investigate the influence on these parameters by illuminating the object with structured light.

The field incident on the object here consists of two fields, i.e:

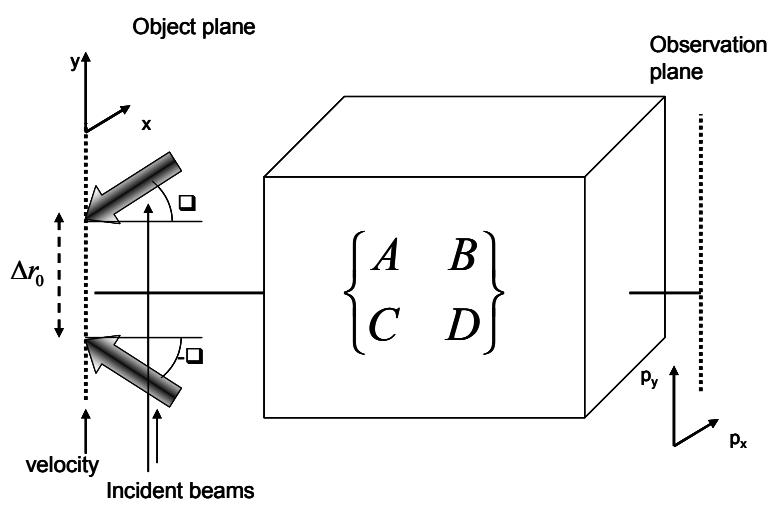

Fig. 1. Illumination of a solid structure with two incident beams, and recording of the speckle pattern after passing an optical $A B C D$-system.

$$
\begin{gathered}
U_{1,2}(\mathbf{r})=U_{0} \exp \left[-\frac{\left(\mathbf{r} \pm \Delta \mathbf{r}_{0} / 2\right)^{2}}{w_{0}^{2}}-\frac{i k\left(\mathbf{r} \pm \Delta \mathbf{r}_{0} / 2\right)^{2}}{2 R_{C}} \mp i k\left(\mathbf{r} \pm \Delta \mathbf{r}_{0} / 2\right) \cdot \theta\right] \\
U_{\text {inc }}(\mathbf{r})=U_{1}(\mathbf{r})+U_{2}(\mathbf{r})
\end{gathered}
$$

The two incident fields have the same $1 / \mathrm{e}^{2}$ intensity radius $\omega_{0}$, radius of curvature $R_{c}$, angle of incidence $\pm \theta$ at their impact centre and a mutual separation $\Delta \mathbf{r}_{0}$. Here, $R_{C}$ is negative (positive) for a divergent (converging) beam. We are now able to track the fields, before and after an object displacement, through the arbitrary optical system by combining the expressions in Eqs. (2), (3), (4), and (7) and inserting this in Eq. (1). In doing this, we would obtain 4 terms for the field correlation in Eq. (6) and 16 terms for the intensity covariance.

As argued in Appendix A, the important contributions to the intensity covariance becomes

$$
\Gamma(\Delta \mathbf{p}, \tau)=\left|\gamma_{11}(\Delta \mathbf{p}, \tau)\right|^{2}+\left|\gamma_{22}(\Delta \mathbf{p}, \tau)\right|^{2}+2 \operatorname{Re}\left[\gamma_{11}(\Delta \mathbf{p}, \tau) \gamma_{22}^{*}(\Delta \mathbf{p}, \tau)\right] .
$$

All mixed terms $\gamma_{12}(\Delta \mathbf{p}, \tau)$ and $\gamma_{21}(\Delta \mathbf{p}, \tau)$ will vanish for the time-scales under consideration here, i.e. we do not consider time-of-flight velocimetry where one probes the time it takes object structures to pass from one illuminated spot to the next.

We are now able to derive the space-time-lagged intensity crosscovariance in the observation plane. For simplicity in presentation, we present below the one-dimensional case where $\Delta \mathbf{p}=(\Delta p, 0)$, and $\Delta \mathbf{r}_{0}=\left(\Delta x_{0}, 0\right)$, where it is tacitly understood that $\mathbf{v}=(v, 0)$ is the component of the peripheral velocity parallel to the $x$-axis. We get: 


$$
\Gamma(\Delta p, \tau)=\exp \left[-\frac{(v \tau)^{2}}{\omega_{0}^{2}}-\frac{\left(\Delta p-v \tau\left[A-B / R_{C}\right]\right)^{2}}{w_{S}^{2}}\right] \cos ^{2}\left[\frac{k \Delta x_{0}}{2 B}\left(\Delta p-v \tau\left(\frac{2 B \theta}{\Delta r}+A\right)\right)\right]
$$

where

$$
w_{S}=\frac{2 B}{k w_{0}} .
$$

For the sake of clarity, we have assumed all matrix elements to be real-valued meaning that the optical system, itself, does not contain absorbing apertures. The addition of apertures is straight-forward by following the above procedure but the results become less intelligible. The speckle size is in Eq. (10) given by $w_{s}$. A closer look at Eq. (9) reveals that the first exponential contribution is the conventional speckle displacement with the following properties:

- $\quad$ speckle size $2 B /\left(k \omega_{0}\right)$

- speckle velocity: $v\left(A-B / R_{C}\right)$

- decorrelation distance in object plane: $\omega_{0}$.

In case of free space propagation a distance $L$, we have $A=1$ and $B=L$, which by insertion in the above expressions shows the well-known speckle parameters. The second cosine-term describes the fine structure that for certain parameters might "dress" the larger speckles with a fringe pattern having an arbitrary phase from one speckle to the next. The following characteristics are associated with this fine structure:

- fringe spacing $B \lambda / \Delta r_{0}$

- fringe velocity $v\left(A+2 B \theta / \Delta r_{0}\right)$

Based on the above, the average number of fringes within a speckle becomes $\Delta r_{0} /\left(\pi \omega_{0}\right)$, which is the relative separation of the incident spots on the object measured in units of their beam diameters. Likewise, the ratio between the fringe velocity and the speckle velocity becomes $\left(A+2 B \theta / \Delta r_{0}\right) /\left(A-B / R_{c}\right)$. It is therefore unlikely that the two patterns will move with identical velocities during the object displacement. In fact, situations may arise where the fringe pattern moves in one direction, and the underlying speckle pattern (watermark!) will move in the opposite direction.

Next, we will summarize the above 5 important parameters for the three generic systems:

- Free space propagation a distance $\mathrm{L}:(\mathrm{A}=1, \mathrm{~B}=\mathrm{L})$

- Imaging with magnification $M:(A=M, B=0)$

- Fourier transformation with object at a distance $f$ from a lens with focal length $f$, and observation in the back focal plane of the lens: $(A=0, B=f)$

We get the following parameters shown in Table 1.

\begin{tabular}{||l|l|l|l|l|l||}
\hline & SPECKLE SIZE & $\begin{array}{l}\text { SPECKLE } \\
\text { VELOCITY }\end{array}$ & $\begin{array}{l}\text { DECORRELATION } \\
\text { IN OBJECT SPACE }\end{array}$ & $\begin{array}{l}\text { FRINGE } \\
\text { SPACING }\end{array}$ & $\begin{array}{l}\text { FRINGE } \\
\text { VELOCITY }\end{array}$ \\
\hline Free Space & $\frac{2 L}{k \omega_{0}}$ & $v\left(1-\frac{L}{R_{c}}\right)$ & $\omega_{0}$ & $\frac{L \lambda}{\Delta r_{0}}$ & $v\left(1+\frac{2 L \theta}{\Delta r_{0}}\right)$ \\
\hline Imaging & $\begin{array}{l}\propto 0 \\
\text { Defined by limiting } \\
\text { aperture }\end{array}$ & $M v$ & $\omega_{0}$ & $\begin{array}{l}\text { non-existing } \\
\text { for } \Delta r_{0}>2 \omega_{0}\end{array}$ & $M v$ \\
\hline $\begin{array}{l}\text { Fourier } \\
\text { transform }\end{array}$ & $\frac{2 f}{k \omega_{0}}$ & $\frac{f v}{R_{c}}$ & $\omega_{0}$ & $\frac{\lambda f}{\Delta r_{0}}$ & $\frac{2 f \theta v}{\Delta r_{0}}$ \\
\hline
\end{tabular}

Table 1. Dynamic parameters for three generic optical systems in case of transverse object velocity $v$.

In case the scattering structure is a rotating shaft with radius $R$, a similar evaluation as above can be conducted. To be precise, the value $R$ is the radius of rotation, which usually coincides with the object radius. We consider out-of-plane 
rotation of the object, i.e. the axis of rotation is perpendicular to the optical axis. This case had previously been discussed when the object is illuminated with structured light ${ }^{17}$. As shown, we can use the above formulas by introducing a first optical element, namely a lens with focal length $-R / 2$, followed by the actual optical system. In doing so, we have the following new parameters to be used in Eq. (9):

$$
A \rightarrow A+2 B / R ; B \rightarrow B, \text { and } v \rightarrow \omega R,
$$

where $\omega$ is the angular velocity of the object. We get the following expressions shown in Table 2 for the space- timecovariance of the speckle pattern for the three generic systems form Table 1.

\begin{tabular}{||l|l|l|l|l|l||}
\hline & SPECKLE SIZE & $\begin{array}{l}\text { SPECKLE } \\
\text { VELOCITY }\end{array}$ & $\begin{array}{l}\text { DECORRELATION } \\
\text { IN OBJECT SPACE }\end{array}$ & $\begin{array}{l}\text { FRINGE } \\
\text { SPACING }\end{array}$ & $\begin{array}{l}\text { FRINGE } \\
\text { VELOCITY }\end{array}$ \\
\hline Free Space & $\frac{2 L}{k \omega_{0}}$ & $\omega R\left(1+\left(\frac{2 L}{R}-\frac{L}{R_{c}}\right)\right)$ & $\omega_{0}$ & $\frac{L \lambda}{\Delta r_{0}}$ & $\omega\left(R+2 L\left(1+\frac{\theta R}{\Delta r_{0}}\right)\right)$ \\
\hline Imaging & $\begin{array}{l}\propto 0 \\
\text { Defined by } \\
\text { limiting aperture }\end{array}$ & $\omega M R$ & $\omega_{0}$ & $\begin{array}{l}\propto 0 \text { for } \\
\Delta r_{0}>\omega_{0}\end{array}$ & $\omega M R$ \\
\hline $\begin{array}{l}\text { Fourier } \\
\text { transform }\end{array}$ & $\frac{2 f}{k \omega_{0}}$ & $\omega f\left(2-\frac{R}{R_{c}}\right)$ & $\omega_{0}$ & $\lambda f / \Delta r_{0}$ & $\omega\left(2 f+\frac{2 f \theta R}{\Delta r_{0}}\right)$ \\
\hline
\end{tabular}

Table 2. Dynamic parameters for three generic optical systems in case of a rotational velocity $\omega$ for a shaft of radius $R$.

If our goal is to establish either an optical system that probes peripheral velocity or rotational speed of a rotating structure, we have to achieve speckle velocities that are either proportional to $\omega R$, or $\omega$ independent of $R$, respectively. Next, we can choose either to use the speckles themselves or the fringe velocity as a measure of the quantity, we intend to measure. Finally, we can choose one of the three generic systems for our purpose. As an example, let's assume we wish to use the fringe velocity in a free space system for probing surface velocity. The associated fringe velocity is found in the right uppermost corner of Table 2 . We then seek the condition for the fringe velocity being proportional to $\omega R$ alone, which becomes $R \gg 2 L \Delta r /\left(\Delta r_{0}+2 L \theta\right)$. Following this path, Table 3 sums up the possibilities for establishing these "clean" setups.

\begin{tabular}{|c|c|c|c|}
\hline$\{$ Measurand $\downarrow\} \quad\{$ Optical system $\rightarrow\}$ & OPTICAL SYSTEM & SPECKLE VELOCITY & FRINGE VELOCITY \\
\hline \multirow[t]{3}{*}{ Surface velocity, $\omega R$ dependence } & Free space & $\begin{array}{l}R>>2 L \wedge R>>\left|2 R_{c}\right| \\
\vee R=2 R_{c}\end{array}$ & $R>>2 L \wedge L \theta>>\Delta r$ \\
\hline & Imaging & No restrictions & No restrictions \\
\hline & Fourier transform & $R>>\left|R_{c}\right|$ & $\Delta r_{0} \gg \theta R$ \\
\hline \multirow[t]{3}{*}{$\begin{array}{l}\text { Rotational velocity, } \\
\omega \text {-dependence }\end{array}$} & Free space & $\begin{array}{l}2 L \gg>R \wedge\left|2 R_{c}\right| \gg>R \\
\vee L=R_{c}\end{array}$ & $2 L \gg>R \wedge \Delta r>>\theta R$ \\
\hline & Imaging & Not possible & Not possible \\
\hline & Fourier transform & $R<<\left|R_{c}\right|$ & $\Delta r_{0} \gg \theta R$ \\
\hline
\end{tabular}

Table 3. Restrictions for establishing optical systems for probing peripheral velocity and rotational speed for rotating objects.

Designing of an optical system for measuring surface displacement can rely either on Spatial Filtering Velocimetry, in which case the fringe velocity has to be probed, or it can rely on correlation of recorded speckle patterns, in which case 
speckle velocity might be appropriate. In the latter case, a collective movement of both patterns might be preferred, in which case Table 1 and 2 help establishing the demands for the illuminating fields.

\section{MEASUREMENTS}

A simple setup showing the effect of creating a fine structure inside speckles embedded in a speckle pattern were created as shown in Fig. 2.

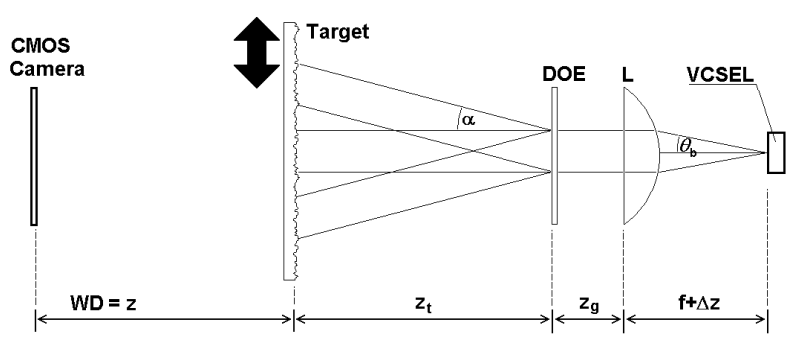

Fig. 2. Setup for probing dynamical behavior of compound speckles

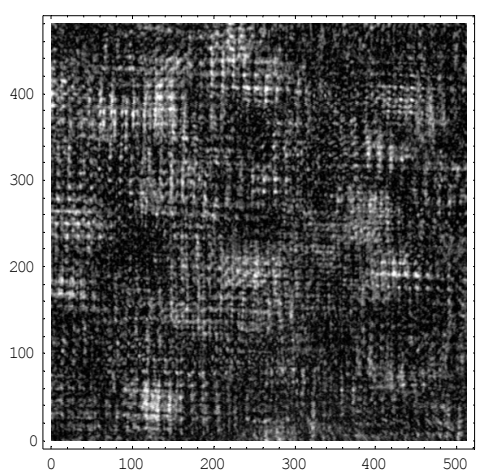

Figure 3. speckle pattern with fine structure.

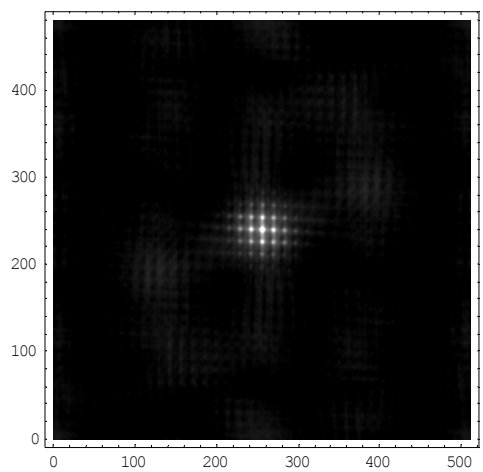

Figure 4. Autocorrelation function of the speckle pattern in Fig. 3.
The light from a Vertical Cavity Surface Emitting Laser (VCSEL) is partly collimated with lens $\mathrm{L}$ and sent through the Diffractive Optical Element (DOE) that here splits up the beam in three angularly separated beams. A diffuse screen is placed at a distance $z_{t}$ from the DOE, and the transmitted field is observed with a CMOS camera at a distance $z$ from the screen. The compound speckle pattern is observed as the screen is laterally displaced between recordings. An example of the observed speckle pattern is shown in Fig. 3, and the associated autocorrelation function is shown in Fig. 4.

A set of experiments were performed with the setup shown in Fig. 2. The following parameters with reference to Tables 1-3 were applicable: $\omega_{0}=70 \mu \mathrm{m}, \Delta r_{0}=570 \mu \mathrm{m}, \theta=3.2^{\circ}$ and $L=60 \mathrm{~mm}$.

We recorded the compound speckle pattern before and after a minor translation of the object. During the postprocessing the speckles and the fine structure were separated by spatial filtering, and finally, the simultaneous displacement of the speckle pattern and the fringe pattern, were measured by crosscorrelation and subsequent peak finding. The gains of the two structures, defined as the ratio between the displacement of the respective structures and the object displacement are shown in Fig. 5 as a function of the off-setting of the VCSEL with respect to back focal plane of the lens $L$. This resulted in a rapid variation of the radii of curvature of the three incident beams, $R_{c}$. 


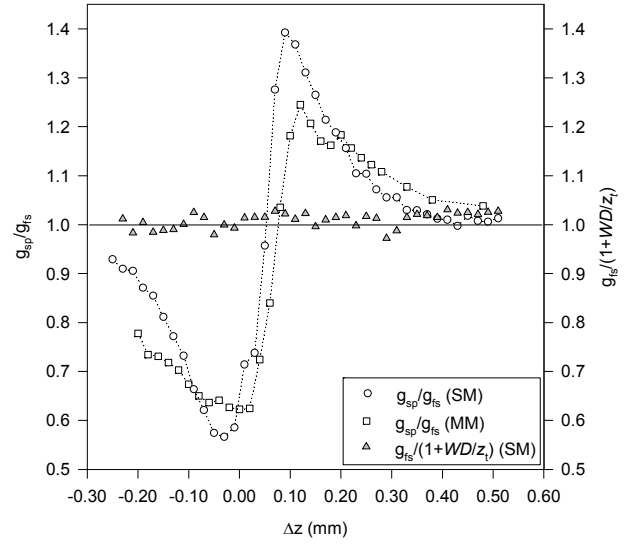

Figure 5. The ratio of the gain factor for the speckle pattern and the gain factor for the fine structure is plotted (circles and squares, left axis) as a function of $\Delta z$. Further, the gain factor of for the fine structure is normalized to the expected gain and plotted (triangles, right axis) as a function of $\Delta \mathrm{z}$ as well. Speckle gain is plotted both for a single (SM) and a multimode VCSEL (MM).
According to Table 1 the speckle velocity will depend on this curvature, whereas the fringe velocity will be independent of $R_{c}$. The speckle displacement were measured both with a single-mode VCSEL and with a multimode VCSEL with a smaller output angle of divergence, resulting in an increased spot size at the object. Although the spot size in itself does not influence the speckle velocity, when scanning the same distance of $\Delta z$ the range of the confocal parameters of the beams incident on the object will be smaller. Accordingly, the range of the radius of curvature of the beams at the object decreases, and this will reduce the change in speckle gain, as shown in Fig. 5.

One of the goals for this investigation is to establish a measurement system for probing rotational speed, where e.g. the speckle velocity uniquely probes the rotational velocity and e.g. the fringe velocity probes the peripheral velocity. Using the result from Table 2, one can see that an ideal optical system cannot be achieved, but in case of a Fourier transform setup, a sufficiently applicable system can be achieved when $R<<R_{c}$ and $\theta R>\Delta r_{0}$.

\section{CONCLUSION}

The influence on the dynamic properties of speckles arising from scattering off solid structures has been investigated in case the object is illuminated with two beams. The object has been assumed to give rise to fully developed speckles, and the two incident beams have arbitrary but equal radius of curvature and hit the object at opposite but arbitrary angles. Further, the distance between the two spot centers is a free parameter, although this separation has been assumed larger that the spot sizes of the incident beams.

It has been shown that the observed speckle pattern possesses the well known speckle characteristics when illumination is with one beam, only. In addition, each speckle is dressed with a fringe pattern with an arbitrary phase change from one speckle to the next. The dynamical behavior of these two structures is in general different, revealing different aspects of the dynamics of the object. Specifically, the dynamics have been studied both for linear and rotational displacement of the object for the three generic optical systems connecting the object and the observation plane, i.e. free space propagation, imaging and Fourier transform.

Finally, expressions have been presented where speckle size, speckle decorrelation length, speckle velocity, fringe spacing and fringe velocity have been found for compound speckles. Expressions were only given for real-valued $A B C D$-matrix elements and the path to derive the results for complex matrix elements was shown. This would provide the results in case the optical system included Gaussian apodized transmission apertures in the $A B C D$-formalism.

\section{ACKNOLEDGMENT}

The present project was financially supported by OPDI Technologies A/S, Denmark. 


\section{APPENDIX A}

The field correlation function Eq. (6) becomes ${ }^{13}$

$$
\gamma(\Delta \mathbf{p}, \tau)=\gamma_{11}(\Delta \mathbf{p}, \tau)+\gamma_{12}(\Delta \mathbf{p}, \tau)+\gamma_{21}(\Delta \mathbf{p}, \tau)+\gamma_{22}(\Delta \mathbf{p}, \tau)
$$

where

$$
\begin{gathered}
\gamma_{i i}(\Delta \mathbf{p}, \tau)=\int d^{2} r G(\mathbf{r}, \mathbf{p}) G^{*}(\mathbf{r}-v \tau, \mathbf{p}-\Delta \mathbf{p}) U_{i}(\mathbf{r}) U_{i}^{*}(\mathbf{r}-v \tau), i=1,2 \\
\quad \text { and } \\
\gamma_{12}(\Delta \mathbf{p}, \tau)=\int d^{2} r G(\mathbf{r}, \mathbf{p}) G^{*}(\mathbf{r}-v \tau, \mathbf{p}-\Delta \mathbf{p}) U_{1}(\mathbf{r}) U_{2}^{*}(\mathbf{r}-v \tau)
\end{gathered}
$$

Here we have taken advantage of the assumption of delta-correlation of the scattering structure, as described in Eq. (4). Substituting Eqs. (9) and (10) into Eq. (6) and rearranging terms yields

$$
\begin{aligned}
\Gamma(\Delta \mathbf{p}, \tau)= & \left|\gamma_{11}(\Delta \mathbf{p}, \tau)\right|^{2}+\left|\gamma_{22}(\Delta \mathbf{p}, \tau)\right|^{2}+2 \operatorname{Re}\left[\gamma_{11}(\Delta \mathbf{p}, \tau) \gamma_{22}^{*}(\Delta \mathbf{p}, \tau)\right]+\left|\gamma_{12}(\Delta \mathbf{p}, \tau)\right|^{2}+\left|\gamma_{21}(\Delta \mathbf{p}, \tau)\right|^{2} \\
& +2 \operatorname{Re}\left[\begin{array}{l}
\gamma_{11}(\Delta \mathbf{p}, \tau) \gamma_{12}^{*}(\Delta \mathbf{p}, \tau)+\gamma_{11}(\Delta \mathbf{p}, \tau) \gamma_{21}^{*}(\Delta \mathbf{p}, \tau)+\gamma_{12}(\Delta \mathbf{p}, \tau) \gamma_{21}^{*}(\Delta \mathbf{p}, \tau) \\
+\gamma_{22}(\Delta \mathbf{p}, \tau) \gamma_{21}^{*}(\Delta \mathbf{p}, \tau)+\gamma_{22}(\Delta \mathbf{p}, \tau) \gamma_{12}^{*}(\Delta \mathbf{p}, \tau)
\end{array}\right]
\end{aligned}
$$

Upon performing the indicated integrations we obtain that the last seven terms on the right hand side of Eq. (A1) (i.e., terms that contain quantities of the form $\gamma_{i j}$, where $\left.i \neq j\right)$ are proportional to either $\exp \left[-\left(\left|\Delta \mathbf{r}_{0}\right|-v \tau\right)^{2} / \omega_{0}^{2}\right]$ or $\exp \left[-\left|\Delta \mathbf{r}_{0}\right|^{2} / \omega_{0}^{2}\right]$. In all cases of practical concern we have that $\tau<<\left|\Delta \mathbf{r}_{0}\right| / v$, and because $\left|\Delta \mathbf{r}_{0}\right|$ is large compared to $\omega_{0}$ these six terms are infinitesimally small and can be neglected. On the other hand, the contribution from the first three terms are finite and yield (for simplicity in presentation we later present the one-dimensional case where $\left|\Delta \mathbf{r}_{0}\right|=2 L \theta$, where it is tacitly understood that $\mathbf{v}$ is the component of velocity parallel to the p- axis). This result is used in Eq. (8).

\section{REFERENCES}

1. J. W. Goodman, Speckle Phenomena in Optics (Roberts \& Company, 2007).

2. M. L. Jakobsen and S. G. Hanson, " Speckle dynamics for intensity-modulated illumination," Appl. Opt. 47, 36743680 (2008).

3. S. G. Hanson, M. L. Jakobsen, R. S. Hansen, and H. T. Yura , "Compound speckles and their statistical and dynamical properties," Proc SPIE Int Soc Opt Eng 7008, 70080M (2008).

4. T. Okamoto and S. Fujita, " Statistical properties of three-dimensional speckle distributions produced by crossed scattered waves," J. Opt. Soc. Am. A 25, 3030-3042 (2008).

5. J. T. Ator , " Image Velocity Sensing by Optical Correlation," Appl. Opt. 5, 1325-1331 (1966).

6. U. Schnell, J. Piot, and R. Dändliker, " Detection of movement with laser speckle patterns: statistical properties," J. Opt. Soc. Am. A 15, 207-216 (1998).

7. H. J. Tiziani , " A study of the use of laser speckle to measure small tilts of optically rough surfaces accurately," Opt. Commun. 5, 271-276 (1972).

8. M. Sjodahl and L. R. Benckert , "Systematic and random errors in electronic speckle photography," Appl. Opt. 33, 7461-7471 (1994). 
9. Y. Aizu and T. Asakura, Spatial filtering velocimetry: Fundamentals and applications (Springer-Verlag, Berlin Heidelberg 2006).

10. T. Asakura and N. Takai, " Dynamic laser speckles and their application to velocity measurements of the diffuse object," Appl. Phys. A 25, 179-194 (1981).

11. Y. Aizu, T. Ushizaka, and T. Asakura, " Measurements of flow velocity in a microscopic region using a transmission grating: elimination of directional ambiguity," Appl. Opt. 24, 636-640 (1985).

12. D. L. Fried , " Laser eye safety: the implications of ordinary speckle statistics and of speckled-speckle statistics." J. Opt. Soc. Am. 71, 914-916 (1981).

13. H. T. Yura, S. G. Hanson, and M. L. Jakobsen, " Speckle dynamics resulting from multiple interfering beams," J. Opt. Soc. Am A, 25, 318-326 (2008).

14. H. T.Yura and S. G. Hanson, " Optical Beam Wave Propagation through Complex Optical Systems,” J. Opt. Soc. Am, 4, 1931-1948 (1987).

15. H. T. Yura, S. G. Hanson, and T. Grum, " Speckle statistics and interferometric decorrelation effects in complex $A B C D$ optical systems," J. Opt. Soc. Am A, 10, 316-323 (1993).

16. H. Yura, S. Hanson, R. Hansen, and B. Rose , " Three-dimensional speckle dynamics in paraxial optical systems," J. Opt. Soc. Am A 16, 1402-1412 (1999).

17. M. L. Jakobsen, H. T. Yura, and S. G. Hanson, " Speckle dynamics for dual-beam optical illumination of a rotating structure," Appl. Opt. 48, 1804-1811 (2009). 